\title{
Hardness and Elasticity of Sound and Caries-affected Primary Dentin Bonded with One-step Self-etch Adhesive
}

\author{
Yumiko HOSOYA ${ }^{1}$, Franklin R. TAY ${ }^{2}$, Masashi MIYAZAKI ${ }^{3}$ and Takashi INOUE ${ }^{4}$ \\ ${ }^{1}$ Department of Pediatric Dentistry, Course of Medical and Dental Sciences, Nagasaki University Graduate School of \\ Biomedical Sciences, 1-7-1, Sakamoto, Nagasaki 852-8588, Japan \\ ${ }^{2}$ Department of Oral Biology and Maxillofacial Pathology, School of Dentistry, Medical College of Georgia, Augusta, GA \\ 30912-1129, USA \\ ${ }^{3}$ Department of Operative Dentistry, Nihon University School of Dentistry, 1-8-13, Kanda-Surugadai, \\ Chiyoda-ku, Tokyo 101-8310, Japan \\ ${ }^{4}$ Department of Clinical Pathophysiology, Tokyo Dental College, 1-2-2, Masago, Mihama-ku, Chiba 261-8502, Japan \\ Corresponding author, Yumiko HOSOYA; E-mail: hosoya@nagasaki-u.ac.jp
}

Received November 7, 2006/Accepted February 16, 2007

Biomechanical properties of bonded dentin are important for resin restorations. We hypothesized that there are no differences in the hardness and elasticity of sound and caries-affected primary dentin bonded with a one-step self-etch adhesive. Resin-dentin interfaces in sound and caries-affected primary dentin were measured with a nano-indentation tester and observed with SEM and TEM. Interfacial dentin hardness was similar for sound and caries-affected dentin, but significantly lower than the underlying intact dentin. As for the Young's modulus of interfacial dentin, both substrates exhibited significantly lower values than the subsurface dentin. Further, the Young's modulus of interfacial dentin was significantly lower in caries-affected dentin. TEM revealed extensive interfacial nanoleakage in bonded sound dentin, while it was minimal in bonded caries-affected dentin. However, in the latter, silver deposits were identified within the porous substrate. Shorter application time and/or improvement of the adhesive components may be required to obtain stable adhesion in primary dentin.

Keywords: Hardness, Young's modulus, Nanoleakage, Caries-affected dentin, Sound dentin

\section{INTRODUCTION}

Resin adhesion to dentin relies on the impregnation of resin into a superficially demineralized dentin zone of exposed collagen fibrils ${ }^{1}$. Previous studies have evaluated the hardness and elasticity of resin-dentin interfaces in permanent teeth using nano-indentation techniques ${ }^{2-4)}$. The hardness of the hybrid layer was significantly lower than that of unaltered dentin, and a gradient of moduli of elasticity was observed from the rather stiff dentin through the elastic hybrid layer and adhesive resin layer ${ }^{2,3}$. It was also found that there were no differences in either the hardness or elasticity of the hybrid layer between sound and carious dentin ${ }^{3)}$.

The hardness ${ }^{5.9)}$ and tensile strength ${ }^{10)}$ of sound primary dentin are lower than sound permanent dentin. Concentrations of calcium and phosphate in both peritubular and intertubular dentin are lower in primary teeth than in permanent teeth ${ }^{11}$. There are also ultrastructural differences between primary and permanent dentin ${ }^{12,13)}$. Differences in the hardness and elasticity of primary and permanent dentin may lead to different characteristics of resin-dentin adhesion. However, information on the hardness and elasticity of bonded primary dentin and their hybrid layers is scarce ${ }^{8,9,14-18}$. Moreover, different results were observed between bonded sound and caries-affected primary ${ }^{17,18)}$ and permanent dentin ${ }^{2-4)}$.

As some of the recently introduced one-step selfetch adhesives produce thin hybrid layers that are less than $1 \mu \mathrm{m}$ thick ${ }^{19)}$. Investigation on the biomechanical properties of these resin-dentin interfaces in sound and caries-affected primary dentin would help to improve our understanding on how dentin bonding affects the physical properties of dentin in primary teeth. Thus, the objectives of this study were two-fold: (1) to evaluate the hardness and elasticity of resin-dentin interfaces and subsurface dentin of sound and carious primary teeth that were bonded with a mild one-step self-etch adhesive; and (2) to examine the ultrastructure of these interfaces. The null hypothesis tested was that there are no differences in hardness and Young's modulus between resin-dentin interfaces created by the adhesive in sound and caries-affected primary dentin.

\section{MATERIALS AND METHODS}

\section{Experimental teeth}

Thirteen primary molars (seven with dentin caries and six non-carious) that were extracted for expediting the eruption of succedaneous tooth were employed in this study. Informed consent was obtained from parents and patients for tooth collection according to the regulations of Nagasaki 
University Dental School (Permission No. 26).

The teeth were frozen in physiologic saline within 10 minutes after extraction. Dentin caries was present on the proximal surfaces of the carious primary molars.

\section{Specimen preparation}

For the sound teeth, flat occlusal dentin surfaces were prepared with a water-cooled high-speed diamond bur. For the carious teeth, the cariesinfected dentin was stained with a caries detector (Caries Check, Nippon Shika Yakuhin, Shimonoseki, Japan ${ }^{20}$ and all the stained dentin was removed with water-cooled low-speed stainless steel round burs.

A single-bottle, one-step self-etch adhesive (Clearfil Tri-S Bond, Kuraray Medical Inc., Kurashiki, Japan) was employed. The major components were MDP (10-methacryloyloxydecyl dihydrogen phosphate), HEMA (2-hydroxyethyl methacrylate), Bis-GMA (bisphenol A diglycidylmethacrylate), silanized colloidal silica, dlcamphorquinone, ethanol and water. The $\mathrm{pH}$ of the adhesive was 2.7. The bonded surfaces were restored with a low-viscosity resin composite (Protect Liner F, Kuraray).

After immersion in distilled water for 24 hours the bonded sound teeth were sectioned buccolingually and parallel to the longitudinal axis at the center of the occlusal surface. The caries teeth were sectioned parallel to the longitudinal axis along the center of the cavity. Sectioning was performed using a low-speed saw (Isomet, Buehler, Lake Bluff, IL) under copious water cooling. After sectioning, the specimens were polished with wet 1200-grit silicon carbide papers. Final polishing was performed with $0.3 \mu \mathrm{m}$ aluminum oxide lapping films (Maruto Co., Tokyo, Japan). The polished specimens were stored in distilled water at $4^{\circ} \mathrm{C}$ until nano-indentation testing.

\section{Nano-indentation test}

One-half of the polished specimens were used for nano-indentation testing (ENT-1100a, Elionix Co., Tokyo, Japan), with a chamber temperature at $26^{\circ} \mathrm{C}$ and humidity at $40-50 \%$. Hardness $(\mathrm{H})$ and Young's modulus (Y) were calculated following procedures as previously described ${ }^{8,9)}$.

Indentations were made at $10 \mu \mathrm{m}$ intervals along the resin-dentin interface, through dentin to pulp chamber wall (Fig. 1) using a load of $300 \mathrm{mgf}$ for 10 seconds each. The data obtained from the first eight indentations of each line were analyzed. Each line of indentations was performed on crack-free locations where the dentinal tubules were perpendicular to the resin-dentin interface. Three lines of indentations were made for each caries-affected dentin specimen, and three to four lines of indentations were made for each sound dentin specimen. The indentations were observed using a microscope with a CCD camera attached to the tester under $\times 700$ magnification. This was to ensure symmetrical indents and adequate spacing between the indentations. Scanning electron microscopy (SEM) was used after argon ion-etching (ERA-8800 FE, Elionix Co.) to eliminate irregularshaped indentations, those contacting the dentinal tubule or located at incorrect portions.

Data were statistically analyzed using one-way analysis of variance and post-hoc Fisher's PLSD at $a=0.05$.

\section{Transmission electron microscopy}

The remaining half of each specimen were fixed with Karnovsky's fixative, embedded in epoxy resin (TAAB 812, TAAB Laboratories, Aldermaston, UK), and prepared for transmission electron microscopy (TEM) using a previously described silver uptake method $^{211}$. One-millimeter-thick slabs were coated with nail varnish applied to within $1 \mathrm{~mm}$ of the bonded interface and immersed in $50 \mathrm{wt} \%$ aqueous ammoniacal silver nitrate $(\mathrm{pH} 9.5)$ for 24 hours. The silver-impregnated slabs were rinsed thoroughly in distilled water and placed in photodeveloping solution

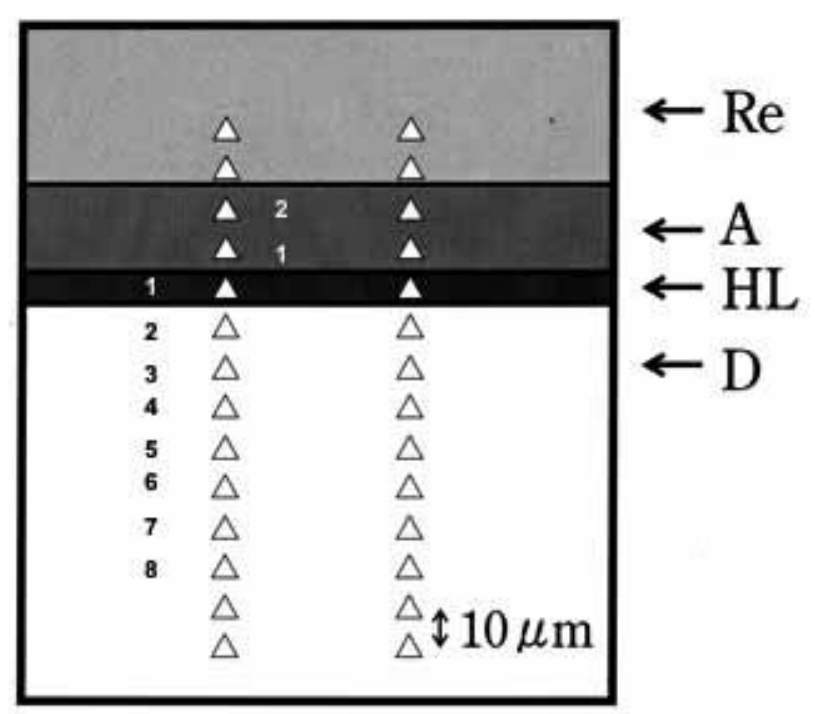

Re : Resin

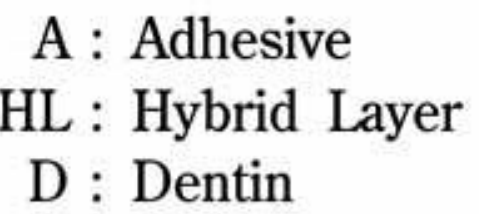

Fig. 1 A schematic of the positions in the nano-indentation test. 
for eight hours under a fluorescent light to reduce the diamine-silver ions into metallic silver grains.

Following dehydration and resin embedding, 90 $\mathrm{nm}$ thick sections containing the resin-dentin interfaces were prepared using an ultramicrotome (Ultracut S, Leica, Vienna, Austria) and a diamond knife. The sections were collected on single-slot, carbon-formvar-coated copper grids (Electron Microscopy Sciences, Fort Washington, PA) and examined without further staining using a TEM (CM100, Philips, Eindhoven, The Netherlands) at 80 $\mathrm{keV}$.

\section{RESULTS}

Table 1 shows the mean hardness $(\mathrm{H})$ and Young's modulus (Y) of caries-affected and sound primary dentin bonded with Clearfil Tri-S Bond. For the carious teeth, the $\mathrm{H}$ value at the interfacial dentin (first indentation) was significantly lower than those obtained from $50 \mu \mathrm{m}$ (point 6) to $70 \mu \mathrm{m}$ (point 8) beneath the interface. There were no significant differences among the $\mathrm{H}$ values of the first five points. For the sound teeth, the $\mathrm{H}$ value at the interfacial dentin was significantly lower than those obtained from $20 \mu \mathrm{m}$ (point 3) to $70 \mu \mathrm{m}$ (point 8) beneath the interface. The $\mathrm{H}$ value at the second point was also significantly lower than those obtained from $40 \mu \mathrm{m}$ (point 5) and $50 \mu \mathrm{m}$ (point 6) beneath the interface. There were no significant differences between the $\mathrm{H}$ values of the first two indentations. For both the carious and sound teeth, the $\mathrm{Y}$ values of the interfacial dentin were significantly lower than those obtained from $10 \mu \mathrm{m}$ to $70 \mu \mathrm{m}$ beneath the interface. There were no significant differences among the $\mathrm{Y}$ values obtained from all the measuring points, except for the first indentation.

Table 2 compares the $\mathrm{H}$ and $\mathrm{Y}$ values between the sound and caries-affected dentin along the same indentations. There were no significant differences between the $\mathrm{H}$ values obtained from caries-affected and sound primary dentin along the interfacial region. However, the $\mathrm{Y}$ value in the interfacial dentin of the bonded caries-affected dentin was significantly lower than that of sound dentin. There was no significant difference of the $\mathrm{H}$ values between the sound dentin and caries-affected dentin at the measuring points of 1,2 and 8 . The $H$ values of the caries-affected dentin were significantly lower than those of sound dentin at the measuring points of 3-7. However, the $\mathrm{Y}$ values of the caries-affected dentin

Table 1 Means and standard deviations of the hardness and Young's modulus of sound and caries-affected primary dentin bonded with Clearfil Tri-S Bond

\begin{tabular}{|c|c|c|c|c|c|}
\hline Dentin & $\begin{array}{l}\text { Measuring } \\
\text { point }\end{array}$ & $\begin{array}{l}\text { Distance } \\
\text { from } \\
\text { interface } \\
(\mu \mathrm{m})\end{array}$ & $\begin{array}{l}\text { Hardness } † \\
\left(\mathrm{mgf} / \mu \mathrm{m}^{2}\right)\end{array}$ & $\begin{array}{c}\text { Young's modulus } \\
\left(\mathrm{mgf} / \mu \mathrm{m}^{2}\right)\end{array}$ & $\begin{array}{l}\text { Number of } \\
\text { measurements }\end{array}$ \\
\hline \multirow{8}{*}{$\begin{array}{l}\text { Caries- } \\
\text { affected }\end{array}$} & 1 & 0 & $42.5 \pm 12.1^{\mathrm{a}}$ & $2012 \pm 685^{\mathrm{a}}$ & 20 \\
\hline & 2 & 10 & $50.7 \pm 14.1$ & $2956 \pm 661^{\mathrm{b}}$ & 21 \\
\hline & 3 & 20 & $49.9 \pm 13.9$ a & $3065 \pm 693^{b}$ & 21 \\
\hline & 4 & 30 & $48.8 \pm 15.2^{\mathrm{a}, \mathrm{b}}$ & $3101 \pm 747^{b}$ & 20 \\
\hline & 5 & 40 & $50.8 \pm 16.2^{\text {а, }}$ & $3271 \pm 769^{\mathrm{b}}$ & 20 \\
\hline & 6 & 50 & $52.4 \pm 17.3^{b}$ & $3399 \pm 912^{b}$ & 21 \\
\hline & 7 & 60 & $53.6 \pm 14.8^{b}$ & $3497 \pm 903^{b}$ & 21 \\
\hline & 8 & 70 & $54.7 \pm 22.2^{b}$ & $3471 \pm 870^{\mathrm{b}}$ & 21 \\
\hline \multirow{8}{*}{ Sound } & 1 & 0 & $48.4 \pm 14.6^{\mathrm{a}}$ & $2734 \pm 890^{a}$ & 20 \\
\hline & 2 & 10 & $56.6 \pm 19.3^{a, c}$ & $3463 \pm 729^{b}$ & 20 \\
\hline & 3 & 20 & $63.6 \pm 14.4^{\mathrm{b}, c}$ & $3683 \pm 627^{b}$ & 20 \\
\hline & 4 & 30 & $60.9 \pm 22.1^{b, c}$ & $3533 \pm 895^{b}$ & 20 \\
\hline & 5 & 40 & $68.2 \pm 23.6^{b}$ & $3841 \pm 927^{b}$ & 20 \\
\hline & 6 & 50 & $68.7 \pm 11.2^{b}$ & $3861 \pm 768^{b}$ & 20 \\
\hline & 7 & 60 & $66.6 \pm 19.0^{b, c}$ & $3803 \pm 963^{b}$ & 20 \\
\hline & 8 & 70 & $65.6 \pm 18.7^{b, c}$ & $3784 \pm 992^{b}$ & 20 \\
\hline
\end{tabular}

$\dagger$ Values are means \pm standard deviations. For the same column within caries-affected or sound dentine, subgroups with same superscripts are no statistically significant difference $(p<0.01)$. 
Table 2 Comparison of means and standard deviations of the hardness and Young's modulus of sound and cariesaffected primary dentin bonded with Clearfil Tri-S Bond

\begin{tabular}{|c|c|c|c|c|c|}
\hline $\begin{array}{l}\text { Measuring } \\
\text { point }\end{array}$ & $\begin{array}{c}\text { Distance } \\
\text { from } \\
\text { interface } \\
(\mu \mathrm{m})\end{array}$ & Dentin & $\begin{array}{l}\text { Hardness } † \\
\left(\mathrm{mgf} / \mu \mathrm{m}^{2}\right)\end{array}$ & $\begin{array}{l}\text { Young's modulus } \\
\left(\mathrm{mgf} / \mu \mathrm{m}^{2}\right)\end{array}$ & $\begin{array}{l}\text { Number } \\
\text { of } \\
\text { measure- } \\
\text { ments }\end{array}$ \\
\hline \multirow{2}{*}{1} & \multirow{2}{*}{0} & Caries-affected & $42.5 \pm 12.1^{\mathrm{a}}$ & $2012 \pm 685$ & 20 \\
\hline & & Sound & $48.4 \pm 14.6^{\mathrm{a}}$ & $2734 \pm 890$ & 20 \\
\hline \multirow{2}{*}{2} & \multirow{2}{*}{10} & Caries-affected & $50.7 \pm 14.1^{\mathrm{b}}$ & $2956 \pm 661$ & 21 \\
\hline & & Sound & $56.6 \pm 19.3^{b}$ & $3463 \pm 729$ & 20 \\
\hline \multirow{2}{*}{3} & \multirow{2}{*}{20} & Caries-affected & $49.9 \pm 13.9$ & $3065 \pm 693$ & 21 \\
\hline & & Sound & $63.6 \pm 14.4$ & $3683 \pm 627$ & 20 \\
\hline \multirow{2}{*}{4} & \multirow{2}{*}{30} & Caries-affected & $48.8 \pm 15.2$ & $3101 \pm 747^{\mathrm{a}}$ & 20 \\
\hline & & Sound & $60.9 \pm 22.1$ & $3533 \pm 895^{a}$ & 20 \\
\hline \multirow{2}{*}{5} & \multirow{2}{*}{40} & Caries-affected & $50.8 \pm 16.2$ & $3271 \pm 769$ & 20 \\
\hline & & Sound & $68.2 \pm 23.6$ & $3841 \pm 927$ & 20 \\
\hline \multirow{2}{*}{6} & \multirow{2}{*}{50} & Caries-affected & $52.4 \pm 17.3$ & $3399 \pm 912$ & 21 \\
\hline & & Sound & $68.7 \pm 11.2$ & $3861 \pm 768$ & 20 \\
\hline \multirow{2}{*}{7} & \multirow{2}{*}{60} & Caries-affected & $53.6 \pm 14.8$ & $3497 \pm 903^{b}$ & 21 \\
\hline & & Sound & $66.6 \pm 19.0$ & $3803 \pm 963^{b}$ & 20 \\
\hline \multirow{2}{*}{8} & \multirow{2}{*}{70} & Caries-affected & $54.7 \pm 22.2^{\circ}$ & $3471 \pm 870^{\circ}$ & 21 \\
\hline & & Sound & $65.6 \pm 18.7^{\circ}$ & $3784 \pm 992^{\circ}$ & 20 \\
\hline
\end{tabular}

$†$ Values are means \pm standard deviations. For each measuring point, no significant differences $(\mathrm{p}<0.01)$ in hardness and Young's modulus between caries-affected and sound dentin are indicated by same superscripts.

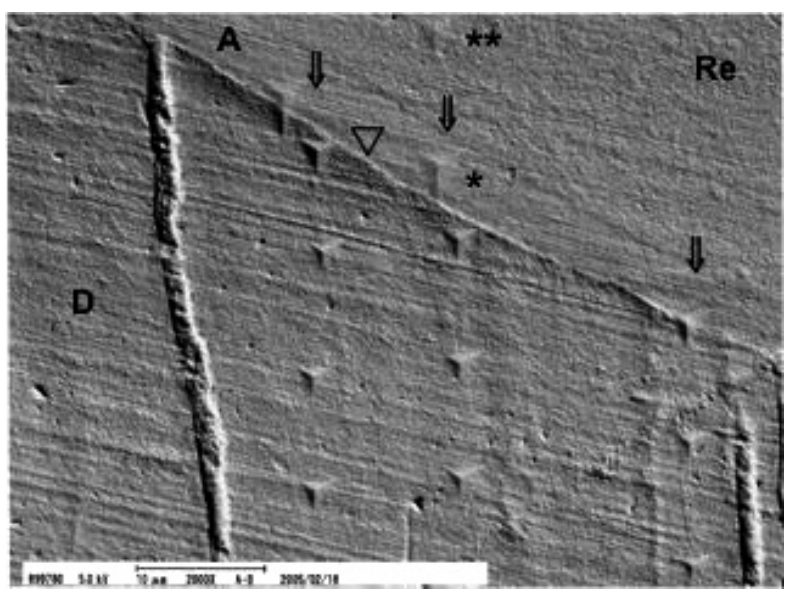

Fig. 2 SEM micrograph of the first few indentations on bonded sound intertubular dentin after argon ion etching. Each arrow indicates an indentation measuring line, and the triangle indicates the hybrid layer. This image was observed with the topographic imaging mode. A thin but distinct hybrid layer was observed beneath the triangle mark. Indentations were observed not only on dentin but also on adhesive (asterisk) and lining resin (double asterisk). D: dentin; A: adhesive; Re: resin composite. were significantly lower than those of sound dentin at the measuring points of $1,2,3,5$ and 6 . There was no significant difference of the $\mathrm{Y}$ values between the sound and caries-affected dentin at the measuring points of 4,7 and 8 .

The $\mathrm{H}$ and $\mathrm{Y}$ values of the adhesive at $10 \mu \mathrm{m}$ above the interface were $26.6 \pm 1.9$ and $1114 \pm 46 \mathrm{mgf} /$ $\mu \mathrm{m}^{2}$ for sound teeth, and $26.7 \pm 2.9$ and $1083 \pm 58$ $\mathrm{mgf} / \mu \mathrm{m}^{2}$ for carious teeth, respectively. These values were significantly lower than those of the underlining dentin $(\mathrm{p}<0.01)$.

Figure 2 depicts the SEM imaging of the first few indentations on bonded sound intertubular dentin after argon ion etching and observed using topographic imaging. Indentations made on the resin-dentin interface that traversed both the dentin and adhesive were deleted from the data.

Transmission electron microscopy (Fig. 3) of silver-impregnated resin-dentin interfaces showed that the hybrid layers were about $1 \mu \mathrm{m}$ thick for both caries-affected and sound dentin. In the sound teeth, both the patent dentinal tubules and the partially demineralized intertubular dentin were filled with extensive silver deposits (Figs. 3A and 3B). 
$[\mathrm{A}]$

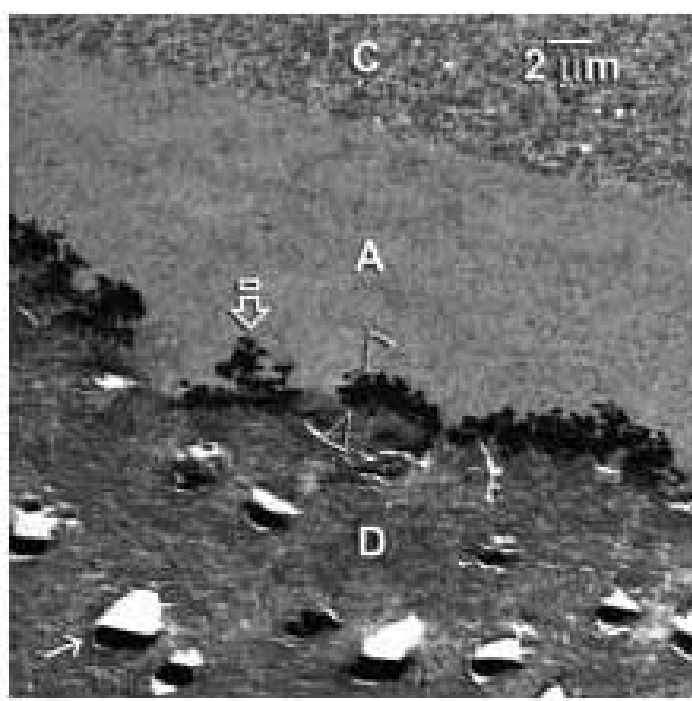

$[B]$

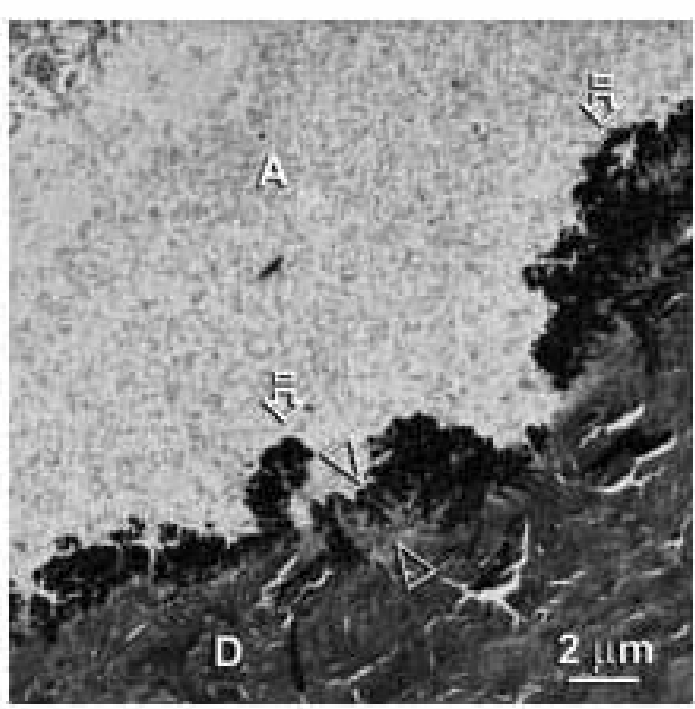

$[\mathrm{C}]$

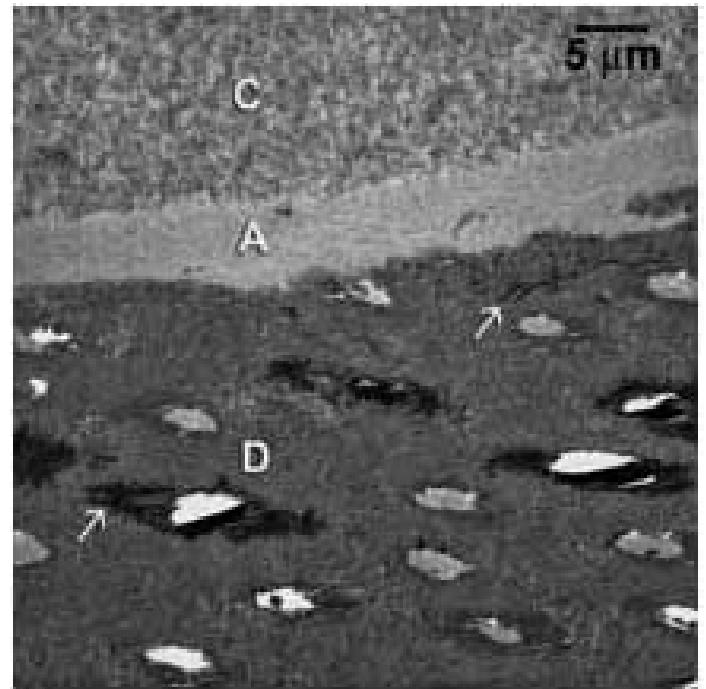

[D ]

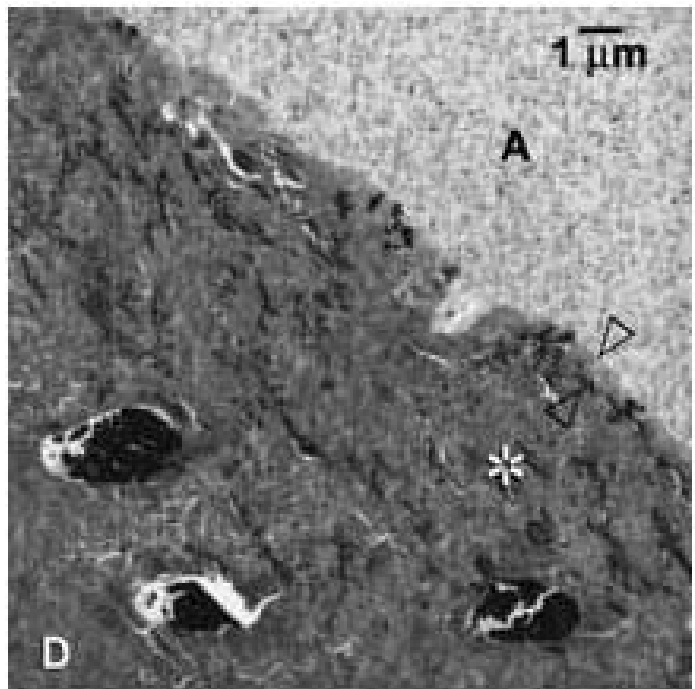

Fig. 3 Representative TEM micrographs obtained from silver-impregnated, unstained and undemineralized sections of resin-dentin interfaces of bonded primary dentin. C: resin composite, A: adhesive, $\mathrm{D}$ : dentin.

(A) A low magnification overall view of the resin-dentin interface from bonded sound primary dentin. The dentinal tubules were filled with silver deposits (arrow), indicating that they were neither infiltrated with the self-etch adhesive nor obliterated by caries crystals. The smear layer was completely dissolved and the self-etch adhesive etched about $0.5-1.5 \mu \mathrm{m}$ into the underlying intact dentin (between open arrowheads). However, almost the entire partially demineralized dentin zone was filled with silver deposits. Some of the silver deposits extended also from the surface of the dentin into the adhesive layer (open arrow).

(B) A high magnification view of Fig. 3A, depicting in detail the previously mentioned features. (C) Resin-dentin interface in caries-affected primary dentin. Some tubules were filled with mineral deposits while other dentinal tubules were patent. There was minimal silver deposit along the dentin surface. However, the porous nature of the caries-affected dentin could be seen by the presence of islands of silver deposits within the carious dentin substrate (arrow).

(D) A high magnification view of another specimen of bonded caries-affected dentin. The dentinal tubule was partially obliterated with crystalline deposits. The overall reduction in fluid transudation resulted in minimal silver nanoleakage within the partially demineralized zone or hybrid layer (between open arrows). However, the zone of caries-affected dentin (asterisk) was porous and contained abundant streak-like silver deposits. 
Conversely, in the carious teeth, some of the dentinal tubules were filled with mineral deposits that prevented silver nanoleakage (Fig. 3C). However, streak-like silver deposits were seen in the underlying porous caries-affected dentin (Fig. 3D).

\section{DISCUSSION}

Previous nano-indentation studies reported that the hardness of the hybrid layer was significantly lower than that of unaltered dentin for sound third molars ${ }^{2,24)}$ and for carious permanent molars ${ }^{3,24}$. Others opined that the hardness of the resin-dentin interface and adhesive resin differ among adhesive systems ${ }^{2526)}$. Although the hardness of the interfacial dentin was lower than that of the underlying intact dentin in some adhesives, these values were not significantly different with other bonding systems ${ }^{25}$. The Young's modulus of the resin-dentin interface has been reported to be significantly lower than that of the underlying unaltered dentin. The elastic moduli significantly decreased in the order of unaltered dentin, hybrid layer, and adhesive resin ${ }^{2}$.

There is limited information on the hardness and elasticity of the resin-dentin interface for primary teeth $^{17,18}$. In previous studies, a two-step self-etching adhesive (Clearfil SE Bond, Kuraray Medical Inc.), a two-step etch-and-rinse adhesive (Single Bond, 3M ESPE, St. Paul, MN, USA), and a one-step self-etch adhesive (One-Up Bond F Plus, Tokuyama Dental, Tokyo, Japan) were used ${ }^{17,18}$. For both carious and sound primary dentin, the hardness of the interfacial dentin was similar to or significantly higher than that of intact dentin beneath the interface. Conversely, the Young's modulus of the interfacial dentin was similar to or significantly lower than the dentin beneath the interface. These results indicated that generally for carious and sound primary teeth, the hardness and Young's modulus of the interfacial dentin were similar to the dentin adjacent to the interface. These results differed from those reported for permanent teeth.

In the present study, the hardness and Young's moduli of the interfacial dentin in both sound and carious primary teeth were significantly lower than the dentin beneath the interface, except for the hardness of the bonded caries-affected dentin (Table 1). These results were similar to those for permanent teeth $^{2,3)}$. Although some of the dentinal tubules in the underlying caries-affected primary dentin were blocked by minerals (Fig. 3C), the caries-affected intertubular dentin was porous (Fig. 3D) - hence the hardness and elastic modulus of this zone could be low. For the sound primary teeth, continuous etching of the intact dentin beneath the hybrid layer by incompletely polymerized acidic resin monomers could also have produced a zone of partially demineralized dentin with sparse resin infiltration ${ }^{27,28)}$. This could have then accounted for the low hardness values obtained from the subsurface dentin at $10 \mu \mathrm{m}$ beneath the interface (Table 1).

For primary dentin, previous studies have shown that areas under carious lesions had significantly lower Knoop hardness than the corresponding areas obtained from sound dentin of these teeth ${ }^{6,77}$. The hardness and Young's modulus under primary carious lesions were also significantly lower than those in sound primary dentin ${ }^{8)}$. In the present study, the results agreed with these previous studies - except for the hardness of the bonded interfacial dentin and the dentin located at $10 \mu \mathrm{m}$ beneath the interface. It was possible that for both carious and sound primary dentin, Clearfil Tri-S Bond could recover the hardness reduction caused by mineral loss, but could not recover the decrease in elastic modulus caused by partial denaturing of the collagen fibrils. This hypothesis has to be tested in the future.

In the present study and a previous study in which one-step self-etch adhesives were used ${ }^{18)}$, the hybrid layer was about $1 \mu \mathrm{m}$ thick. As the first indentation might have included the hybrid layer as well as resin tags and dentin beneath the hybrid layer (Fig. 2), this could have resulted in the large standard deviations of data recorded from the interfacial region of these teeth. Many of the currently available one-step self-etch adhesives produce thin hybrid layers that are less than $1 \mu \mathrm{m}$ thick on permanent dentin ${ }^{19)}$. Thus, the physical properties of both the hybrid layer and subsurface dentin are important to achieve optimal adhesion. Variations exist in the hardness and elastic modulus of dentin obtained from different intra-tooth locations as well as from tooth to tooth ${ }^{8}$. These variations could have additionally contributed to the large standard deviations obtained.

The hardness and elasticity of the resin-dentin interface may also be influenced by the physical properties of the adhesive resins and the efficacy of etching and priming. ${ }^{29}$. Nevertheless, the results of this study and those of previous studies suggested that primary dentin had significantly lower hardness and Young's modulus than permanent dentin ${ }^{9}$, and that primary dentin might be more porous than permanent dentin ${ }^{11,12)}$. Previous reports ${ }^{30-32}$ suggested that primary dentin was more susceptible to acid etching, and shorter etching time for primary dentin was recommended ${ }^{30)}$.

\section{CONCLUSIONS}

Within the limits of the experimental methods employed in this study, we have to partially reject the null hypothesis that there are no differences in 
hardness and Young's modulus between the interfaces created by a mild one-step self-etch adhesive in sound and caries-affected primary dentin. Application time and components of contemporary self-etch adhesives are designed based on data obtained using human or bovine permanent teeth. Thus, shorter application time for primary dentin and/or improvement of the adhesive components may be required to obtain stable adhesion in primary sound and caries-affected dentin.

\section{ACKNOWLEDGEMENTS}

This study was supported by Central grant sponsor: Japanese Society for the Promotion of Science (No. 18592242). The authors also hereby acknowledge the contributions of Mr. Takuji Ito and Mr. Takahiko Uematsu (Elionix Co., Japan) for their technical assistance in operating the nano-indentation tester and field emission scanning electron microscope, and Dr. Shinich Arita for collecting teeth.

\section{REFERENCES}

1) Nakabayashi N, Kojima K, Masuhara E. Effect of HEMA on bonding to dentin. Dent Mater 1992; 8: 125-130.

2) Van Meerbeek B, Willems JP, Celis G, Roos JR, Braem M, Lambrechts P, Vanherle G. Assessment by nano-indentation of the hardness and elasticity of the resin-dentin bonding area. J Dent Res 1993; 72: 14341442.

3) Sugizaki S, Hanaoka K. Evaluation of hybrid layer at the resin/dentin adhesive interface. Jpn J Conserv Dent 2002; 45: 413-427.

4) Oliveira SSA, Marshall SJ, Habelitz S, Gansky SA, Wilson RS, Marshall GW Jr. The effect of a selfetching primer on the continuous demineralization of dentin. Eur J Oral Sci 2004; 112: 376-383.

5) Johnsen DV. Comparison of primary and permanent teeth. In: Oral Development and Histology, $2^{\text {nd }} e d$, Avery JK (ed.), Thiema Medical Publishers, New York, 1994, p.287.

6) Hosoya Y, Marshall SJ, Watanabe LG, Marshall GW Jr. Microhardness of carious deciduous dentin. Oper Dent 2000; 25: 81-89.

7) Hosoya Y, Ono T, Marshall GW Jr. Microhardness of carious primary canine dentin. Pediatr Dent $\mathrm{J}$ 2002; 12: 91-98

8) Hosoya Y, Marshall GW Jr. The nano-hardness and elastic modulus of carious and sound primary canine dentin. Oper Dent 2004; 29: 142-149.

9) Hosoya Y, Marshall GW. The nano-hardness and elastic modulus of sound deciduous canine dentin and young premolar dentin - Preliminary study. J Mater Sci Mater Med 2005; 16: 1-8.

10) Hosoya Y, Kawada E, Liu J, Oda Y, Marshall GW Jr. Microtensile strength of sound primary second molar dentin. J Mater Sci Mater Med 2005; 40: 61816186.

11) Hirayama A. Experimental analytical electron microscopic studies on the quantitative analysis of elemental concentrations in biological thin specimens and its application to dental science. Shikwa Gakuho 1990; 90: 1019-1036.

12) Sumikawa DA, Marshall GW, Gee L, Marshall SJ. Microstructures of primary tooth dentin. Pediatr Dent 1999; 21: 439-444.

13) Koutsi V, Noonan RG, Homer JA, Simpson MD, Mathews WG, Pashley DH. The effect of dentin depth on the permeability and ultrastructure of primary molars. Pediatr Dent 1994; 16: 29-35.

14) Mahoney E, Holt A, Swain M, Kilpatrick N. The hardness and modulus of elasticity of primary molar teeth: An ultramicro-indentation study. J Dent 2000; 28: $589-594$.

15) Angker L, Swain MV, Kilpatrick N. Micromechanical characterization of the properties of primary tooth dentin. J Dent 2003; 31: 261-267.

16) Angker L, Swain MV, Kilpatrick N. Characterising the micro-mechanical behaviour of the carious dentine of primary teeth using nano-indentation. J Biomechanics 2005; 38: 1535-1542.

17) Hosoya Y. Hardness and elasticity of bonded carious and sound primary tooth dentin. J Dent 2006; 34: 164-171.

18) Hosoya Y, Tay FR. Hardness, elasticity and ultrastructure of bonded sound and caries-affected primary tooth dentin. J Biomed Mater Res B Appl Biomater 2007; 81B: 135-141.

19) Sato M, Miyazaki M. Comparison of depth of dentin etching and resin infiltration with single-step adhesive systems. J Dent 2005; 33: 475-484.

20) Hosoya Y, Taguchi T, Tay FR. Evaluation of a new caries detecting dye for primary and permanent carious dentin. J Dent 2007; 35: 137-143.

21) Tay FR, Pashley DH, Yoshiyama M. Two modes of nanoleakage expression in single-step adhesives. J Dent Res 2002; 81: 472-476.

22) Akimoto N, Momoi Y, Takamizu M, Kohno A. Evaluation of microhardness on resin impregnated layer and its surrounding area by nano-indentation. Jpn J Conserv Dent 1996; 39: 556-562.

23) Yokoyama G, Akimoto N, Kohno A. Nano-hardness of resin-dentin bonded area with wet bonding system. Jpn J Conserv Dent 1993; 42: 1167-1172.

24) Yokoyama G. The influence of carious dentin on bond. Jpn J Conserv Dent 2000; 43: 1256-1270.

25) Nakazawa Y, Seino E, Ushiki T, Ogata T, Hirai Y, Kawada E, Oda Y. Microhardness evaluations of resin-dentin bonding areas by nano-indentation. Bull Tokyo Dent Coll 1999; 40: 47-54.

26) Goto S. A study on light-cured resins — The correlation between nano-hardness of dentin-resin interface and dentin bond strength. Jpn J Conserv Dent 1999; 42: 900-908.

27) Carvalho RM, Chersoni S, Frankenberger R, Pashley $\mathrm{DH}$, Prati C, Tay FR. A challenge to the conventional wisdom that simultaneous etching and resin infiltration always occurs in self-etch adhesives. Biomaterials 2005; 26: 1035-1042.

28) Wang Y, Spencer P. Continuing etching of an all-inone adhesive in wet dentin tubules. J Dent Res 2005; 84: 350-354.

29) Hosoya Y, Kamasaki Y, Horiuchi A, Nishiguchi M, Goto G, Marshall GW Jr. Evaluation of dentin preparation systems for resin adhesion. Pediatr Dent J 1999; 9: 9-18. 
30) Olmetz A, Oztas N, Basak F. Comparison of the resin-dentin interface in primary and permanent teeth. J Clinical Pediatr Dent 1998; 22: 293-298.

31) Hosoya Y, Kawashita Y, Marshall GW Jr, Goto G. Influence of Carisolv ${ }^{\mathrm{TM}}$ for resin adhesion to sound human primary dentin and young permanent dentin.
J Dent 2001; 29: 163-171.

32) Hosoya Y, Shinkawa H, Marshall GW. Influence of Carisolv $^{\mathrm{TM}}$ for resin adhesion to sound human primary dentin and young permanent dentin using two different adhesive systems. J Dent 2005; 33: 283-291. 